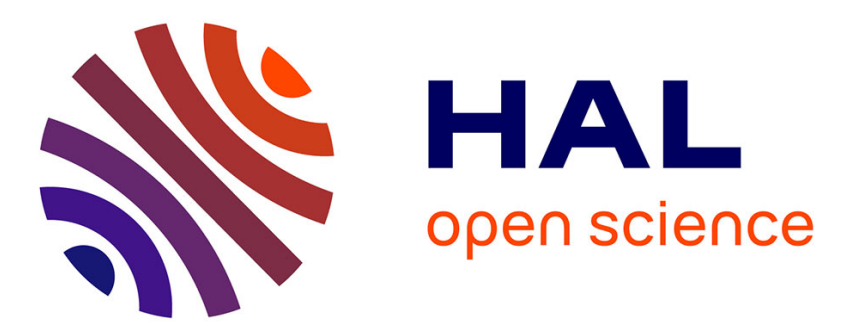

\title{
Multiresponsive Nonvolatile Memories Based on Optically Switchable Ferroelectric Organic Field-Effect Transistors
}

Marco Carroli, Alex G. Dixon, Martin Herder, Egon Pavlica, Stefan Hecht, Gvido Bratina, Emanuele Orgiu, Paolo Samorì

\section{To cite this version:}

Marco Carroli, Alex G. Dixon, Martin Herder, Egon Pavlica, Stefan Hecht, et al.. Multiresponsive Nonvolatile Memories Based on Optically Switchable Ferroelectric Organic Field-Effect Transistors. Advanced Materials, 2021, 33 (14), pp.2007965. 10.1002/adma.202007965 . hal-03194966

\section{HAL Id: hal-03194966 https://hal.science/hal-03194966}

Submitted on 9 Apr 2021

HAL is a multi-disciplinary open access archive for the deposit and dissemination of scientific research documents, whether they are published or not. The documents may come from teaching and research institutions in France or abroad, or from public or private research centers.
L'archive ouverte pluridisciplinaire HAL, est destinée au dépôt et à la diffusion de documents scientifiques de niveau recherche, publiés ou non, émanant des établissements d'enseignement et de recherche français ou étrangers, des laboratoires publics ou privés. 


\section{WILEY-VCH}

DOI: $10.1002 /($ please add manuscript number $))$

\section{Article type: Communication}

Multiresponsive Non-Volatile Memories based on Optically Switchable Ferroelectric Organic Field-effect Transistors

Marco Carroli, Alex G. Dixon, Martin Herder, Egon Pavlica, Stefan Hecht, Gvido Bratina, Emanuele Orgiu, * and Paolo Samori*

Dr. M. Carroli, Prof. E. Orgiu, Prof. P. Samorì

Université de Strasbourg, CNRS, ISIS,

8 alleé Gaspard Monge,

67000 Strasbourg, France

E-mail: samori@unistra.fr

Dr. A.G. Dixon, Dr. E. Pavlica, Prof. G. Bratina

Laboratory of Organic Matter Physics,

University of Nova Gorica,

Vipavska 13,

SI-5000 Nova Gorica, Slovenia

Dr. M. Herder, Prof. S. Hecht

Department of Chemistry \& IRIS Adlershof

Humboldt-Universität zu Berlin

Brook-Taylor-Str. 2, 12489 Berlin, Germany.

Prof. S. Hecht

DWI - Leibniz Institute for Interactive Materials \& Institute of Technical and Macromolecular Chemistry,

RWTH Aachen University, 52074 Aachen, Germany

Prof. E. Orgiu

INRS-Centre Énergie Matériaux Télécommunications 1650 Blv. Lionel-Boulet, J3X 1S2 Varennes, Quebec, Canada

E-mail: emanuele.orgiu@emt.inrs.ca

Keywords: multiresponsive devices, molecular switches, organic electronics, photochromic molecules, ferroelectricity 


\section{WILEY-VCH}

Abstract:

Organic transistors are key elements for flexible, wearable, and bio-compatible logic applications. Multiresponsivity is highly sought-after in organic electronics to enable sophisticated operations and functions. Such challenge can be pursued by integrating in a single device more components, each one responding to a specific external stimulus. Here we report the first multiresponsive organic device based on a photochromic-ferroelectric organic fieldeffect transistor which is capable to operate as non-volatile memory with 11-bit memory storage capacity in a single device. The memory elements can be written and erased independently by means of light or electric field, with accurate control over the readout signal, excellent repeatability, fast response and high retention time. Such a proof-of-concept paves the way towards enhanced functional complexity in opto-electronics via the interfacing of multiple components in a single device, in a fully integrated low-cost technology compatible with flexible substrates. 


\section{WILEY-VCH}

The ubiquity of information technologies has led to the generation of colossal amounts of data, which must be stored and retrieved for very long time. ${ }^{[1]}$ To address the need for an increasingly larger storage capacity in smaller and lightweight flexible devices two approaches can be pursued. One relies on the use of cumbersome lithography-based nanofabrication through which more and more memory cells per unit area can be integrated. ${ }^{[2]}$ The other involves the development of memory cells with increased storage capabilities based on multilevel memories. ${ }^{[3]}$ The latter requires to exploit molecular materials which can be made multiresponsive and multifunctional by design. ${ }^{[4]}$ While conventional memories rely on the use of electrical/magnetic writing and reading, the exploitation of alternative mechanisms to write and read information can impart improved operational robustness to the device. In this framework, a photo-writable/erasable memory in which the electrical readout can be optically programmed promises faster write/erase and a more reliable readout. ${ }^{[5]}$

A simple way to implement multiresponsiveness in a single organic-based device consists in combining different components, each of them imparting a response to a distinct stimulus, in a novel hybrid material and device thereof. ${ }^{[6]}$ Recently, the incorporation of stimuli-responsive molecules in semiconducting organic matrices enabled to add a specific function to solutionprocessed organic field-effect transistors (OFET) ${ }^{[7]}$ In this regard, photoswitchable molecules offering the possibility of optical remote control constitute the ideal functional components for integration into the next-generation opto-electronic memory devices. ${ }^{[8]}$ Among various photoswitchable compounds, diarylethenes exhibit fast photoswitching, high fatigue resistance, thermal bistability, and energy levels that can be tailored through molecular design. ${ }^{[9]}$ Another powerful way to implement data storage in a multilevel memory relies on exploiting the ferroelectricity of a gate insulator in an OFET. ${ }^{[10]}$ One of the most promising organic ferroelectric (insulating) materials is poly(vinylidene fluoride-trifluoroethylene) (PVDF-TrFE) which offers several advantages over conventional organic insulators, ${ }^{[11]}$ most importantly the relatively large fatigue-free remnant polarization, ${ }^{[12]}$ short switching time, and good thermal 


\section{WILEY-VCH}

stability. ${ }^{[13]}$ The number of memory states in a ferroelectric memory cell can be increased by using intermediate polarization states, obtained by applying an external electric field with a magnitude slightly higher than the ferroelectric coercive field. ${ }^{[14]}$ Although ferroelectric polymers and photoswitchable molecules have completely different properties, one should in principle be able to include both components in a suitable device architecture to control the current readout (called drain current) of an OFET in a non-volatile way. Combining their independent operating mechanisms would allow for write and erase operations triggered by different stimuli in the very same memory device.

Here, we demonstrate that fast and robust optical/electrical, multi-level, non-volatile memory operations can be achieved in a multiresponsive optically switchable ferroelectric organic FET (OSFeOFET). In such device, the active layer includes poly(3-hexylthiophene) (P3HT) and a specific diarylethene derivative (DAE-Me). OFETs using such a bicomponent active layer exhibit outstanding photoswitchable capability with the two states being extremely stable over the timescale of several months. ${ }^{[15]}$ In our devices, data are stored in a micrometric transistor memory cell composed of a diarylethene semiconductor blend film placed underneath a ferroelectric gate insulator. Information can be written (erased) into the memory cell independently by either using UV and green light irradiation, respectively, or gate voltage sweeps, while the readout of the stored information is carried out by measuring the drain current.

The geometry of our memory cell and the operating building blocks are shown schematically in Figure 1. The storage of information upon changing the transistor output current occurs through a polarization change in the ferroelectric layer (blue region) or by photoactivated trapping and de-trapping in the photochromic/polymer blend (red region). 


\section{WILEY-VCH}

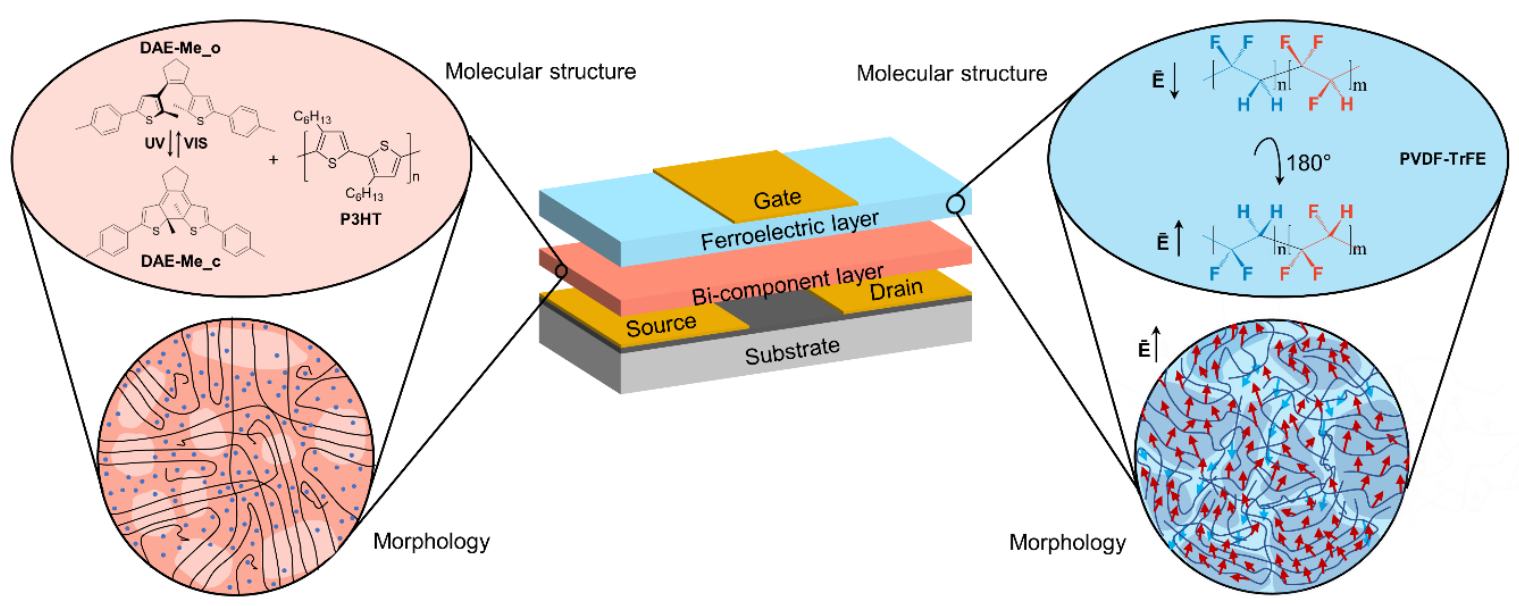

Figure 1: Schematic layout of an optically switchable ferroelectric organic FET (OSFeOFET) comprising a ferroelectric PVDF-TrFE layer (in blue) as gate insulator and a bicomponent P3HT/DAE film (in red) as semiconducting layer. (Left) Photoswitchable P3HT/DAE film: The coexistence of ordered (lighter shaded areas) and amorphous polymer regions allows DAE molecules (blue dots) to be dispersed within the polymer matrix. The "traffic of charges" moving through the bicomponent active film can be controlled with light by locally generating traps. (Right) Ferroelectric polymer layer: PVDF-TrFE polymer chains can undergo a $180^{\circ}$ rotation depending on the intensity of the electric field applied across the dielectric. The morphology influences the capability of the thin film to retain polarization. The ferroelectric $\beta$-crystal-phase of PVDF-TrFE is represented as darker blue shaded area in the morphology sketch. Negative remanent polarization induces a steady (gate) bias within the semicondutor bi-component layer. The device is fabricated on a $\mathrm{Si} / \mathrm{SiO}_{2}$ substrate and the gate, source and drain electrodes are made of gold.

On the one hand, irradiation of the device with UV light triggers the DAE isomerization from the open (DAE-Me_o) to the closed (DAE-Me_c) form, the latter acting as hole acceptor in the semiconducting polymer matrix, thereby lowering the net output current. Hence, data can be written by means of short and low power optical pulses and are encoded in the amount of traps present within the channel. ${ }^{[15]}$ The full reversibility of this isomerization can be achieved upon irradiating the closed form of DAE with green light to reconvert the molecule into its initial open state, thereby erasing the previously stored data. On the other hand, the ferroelectric state 


\section{WILEY-VCH}

of the gate insulator layer influences the surface potential at the interface with the semiconductor and therefore the accumulation/depletion state of the charge in the device. In this case, writing or erasing information is achieved by gate voltage sweeps inducing polarization switching. If the voltage applied is high enough to exceed the coercive field $\left(\mathrm{E}_{\mathrm{c}}\right)$, the dipoles in the ferroelectric layer partially align with the external electric field $\left(E_{0}\right)$ resulting in a permanent polarization after the removal of $\mathrm{E}_{0}$. The magnitude of the polarization vector depends on the difference, i.e. $\left|E_{0}-E_{c}\right|$. This enables the device to reach several partial polarization states which encode multi-level current states. Based on our concept, the three distinct key aspects of Optically Switchable Ferroelectric Organic Field-Effect Transistor (OSFeOFET) include: (1) write and erase cycles of partial non-volatile ferroelectric induced states, (2) light response at full polarization state, and (3) current modulation induced by light on several polarization states, as detailed below.

First, we demonstrate that the OSFeOFET conductivity can be modulated in a non-volatile way by partial polarization of the ferroelectric layer. If the ferroelectric layer is negatively polarized, it can induce a permanent (accumulation) charge layer in the semiconducting film. This means that when a non-zero drain voltage is applied, a drain current can be measured even if the gate voltage is zero. The electrical programming of the fabricated OSFeOFETs was performed by applying an electrical bias sweep between the gate electrode and the source and drain electrodes. The applied erase-write voltage waveform, which is better described in Figure S4, extends from $+40 \mathrm{~V}$ to $\mathrm{V}_{\mathrm{p}}$ (the negative voltage peak) at sweep rate of approximately $2 \mathrm{~V} / \mathrm{s}$. Therefore, depending on $\mathrm{V}_{\mathrm{p}}=-40 \mathrm{~V},-20 \mathrm{~V},-17.5 \mathrm{~V},-15 \mathrm{~V}$ or $-10 \mathrm{~V}$, the resulting P/E cycle time $t_{p-F e}$ ranges from $30 \mathrm{~s}$ to $60 \mathrm{~s}$. Figure 2 shows that five different partial polarization states can be achieved. 


\section{WILEY-VCH}

a)

b)

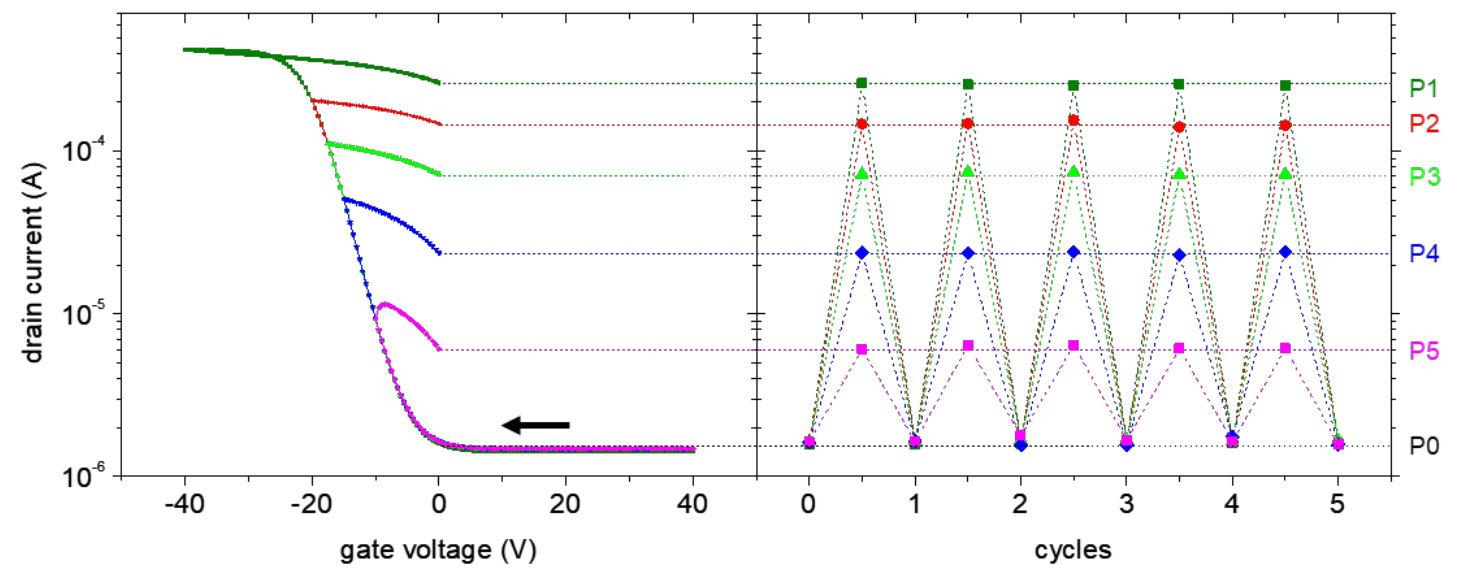

c)

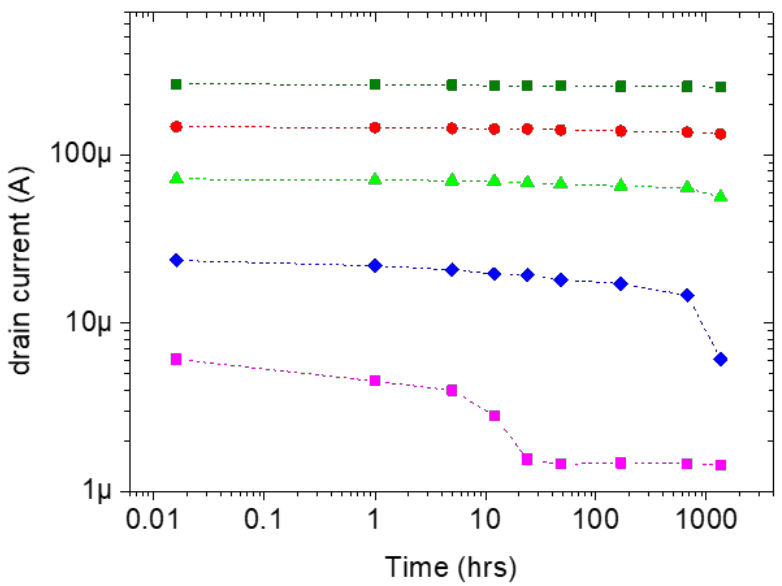

Figure 2: Polarization states and cyclability in P3HT/DAE-Me OSFeOFET. a) Gate voltage sweeps program the ferroelectric OSFeOFET into six non-volatile drain current states P0-P5. The writing process starts from $+40 \mathrm{~V}$ (in order to erase previous polarization state) and sweeps to negative polarization higher than the coercive field: $-40 \mathrm{~V},-20 \mathrm{~V},-17.5 \mathrm{~V},-15 \mathrm{~V}$ and $-10 \mathrm{~V}$ and then returns to $0 \mathrm{~V}$. This current state is now non-volatile programmed in the memory until an erase cycle is performed. It can be measured by applying a drain-source voltage as small as -0.5 V. b) Over several P/E cycles, no fatigue was detected. c) Data retention of P1-P5 current states. At time zero, five different OSFeOFET were programmed according in one of the five polarization cycle described in Figure S4 and the readout current was measured over several days. [Please note that drain current is a hole current and, as such, its sign is negative. As a logarithmic scale is used to emphasize the current change over orders of magnitude, the absolute value of $I_{D}$ was used]

Each of these states corresponds to a different accumulation condition of the FET channel and, therefore, to a distinct FET operating point. The obtained distinct drain current 


\section{WILEY-VCH}

levels (P1, P2, P3, P4, P5) are repeatable over several cycles and no fatigue is observed, as reported in Figure 2b. No fatigue was observed over 5 cycles in the polarization states when a low sweep rate $(\mathrm{V} / \mathrm{s})$ was used in the memory programming phase. In spite of the intrinsic variability of spin-coating process and surface roughness of PVDF-TrFE layer which can affect device reproducibility, ${ }^{[16]}$ PVDF-TrFE devices operated at low frequency showed a fatigue-free polarization over $10^{7}$ cycles. ${ }^{[17]}$ Note that by increasing the P/E cycle frequency would have led to damage our devices' capability irreversibly hindering any further possible tests and analysis. However, to prevent metal delamination and improve the fatigue endurance at higher frequency, several approaches have been reported, ${ }^{[12,17-18]}$ which are fully compatible with the fabrication method of our OSFeOFETs, therefore they could be exploited for lowering the $t_{p-F e}$ range. Although we are aware of the complex nature of the semiconductor/PVDF-TrFE interface and all deposition techniques to optimize it, ${ }^{[11]}$ the scope of the present work is to provide a proof of concept of the potential offered by the combination of ferroelectricity and optical responsivity in the same device.

The last step of the electrical characterization for ferroelectric induced properties in OSFeOFET requires data retention tests to prove that such devices can be used in memory applications. Figure 2c shows that each current level is stable over several hours' time scale. The readout operation of the drain current has been repeated nine times to test the device capability to retain data. Figure 2c shows that the drain current readouts of P1-P5 levels. However, before drawing any conclusion, one should consider that each time the readout is affected by the measurement itself. The read operation consists in probing the drain current level while applying $-0.5 \mathrm{~V}$ as drain-source voltage. Since the gate and source are both connected to the ground, the $\mathrm{V}_{\mathrm{DS}}$ between source and drain locally perturbs the dipole alignment of the ferroelectric layer, resulting in a lowering of the ferroelectric polarization. ${ }^{[19]}$ Corrected data are shown in Figure S6. 


\section{WILEY-VCH}

After demonstrating its working principle, we took our memory concept a step further by illustrating how the multi-bit ferroelectric device can be controlled by light. Our approach relies on the wavelength-dependent composition of the photoswitchable semiconducting blend, which enables wavelength-selective control on the current of non-volatile memory elements (as demonstrated previously for simple blend, optically switchable memories with a storage 'lifetimes' exceeding 500 days $^{[15]}$ ). Figure 3a shows the change in output current upon repeated switching from DAE's open state (interacting with the HOMO of P3HT only electrostatically) to closed state (with DAE acting as hole trap). Exposure to $310 \mathrm{~nm}$ and $530 \mathrm{~nm}$ light enables to decrease and increase the device output current, respectively, thus accessing different memory states. The results demonstrate unequivocal multi-level data storage in our photoswitchable memory transistor, with good signal to noise ratio (SNR). P3HT was chosen as the semiconductor as the presence of DAEs in this particular polymer matrix was found not to disrupt its crystallinity. ${ }^{[20]}$ As illustrated in Figure 3b, the switching ratio is proportional to UV light irradiation time and it is highly reproducible with a measured confidence interval of $\pm 0.5 \%$. In order to test the device limit, the fabricated transistor memory elements are operated using a transient photoconductivity setup as described in the Supplementary Materials. Writing $(\lambda=310 \mathrm{~nm})$ and erasing $(\lambda=530 \mathrm{~nm})$ are performed with nanosecond light pulses generated with a laser. As alternative the erase process is also completed by means of a green LED with the same wavelength. The energy of both writing and erasing pulses is precisely controlled using a set of reflective filters. The initial high-conductivity state (Level P1) in Figure 3c is prepared by fully negatively polarizing the ferroelectric layer and fully opening the DAE molecules, in such a way that the maximum number of current states is ensured. The absolute current variation per laser pulse is determined by the laser pulse energy employed, which defines the amount of DAE molecules that switches inside the blend. To ensure high modulation between the open and closed states we use a transistor of $20 \mu \mathrm{m}$ channel length and $10 \mathrm{~mm}$ channel width. With this device, a change in current readout of $1 \%$ is achieved using a single 


\section{WILEY-VCH}

3-ns write pulse of $2.17 \cdot 10^{-7} \mathrm{~J} \mathrm{~cm}^{-2}$. The DAE open-to-closed photoisomerization exhibits a nearly 50 times higher quantum yield than the reverse, closed-to-open photoisomerization. ${ }^{[21]}$ This conveniently allows the use of pulses with lower incident pulse energy when switching from open to closed states than for the opposite direction. Initially the pulses induce isomerization of the DAE molecules closer to the interface between the semiconductor and ferroelectric layers, where the majority of mobile charge pathways reside, inducing a higher current drop per laser pulse (see inset 1 Figure 3c). Therefore, the number of DAE-Me_o in the channel will progressively diminish after each pulse and the current variation will decrease and reach saturation over $\sim 500$ irradiation pulses, as shown in Figure 3c. In total, more than 500 current variations are distinguishable for each ferroelectric polarization state, yielding to a total amount of 2500 "levels" (>11-bit) if one considers the five ferroelectric polarization states. For the optical erase process to go back to Level P1, a continuous green light irradiation is used, as described in the Supporting Materials. 
WILEY-VCH
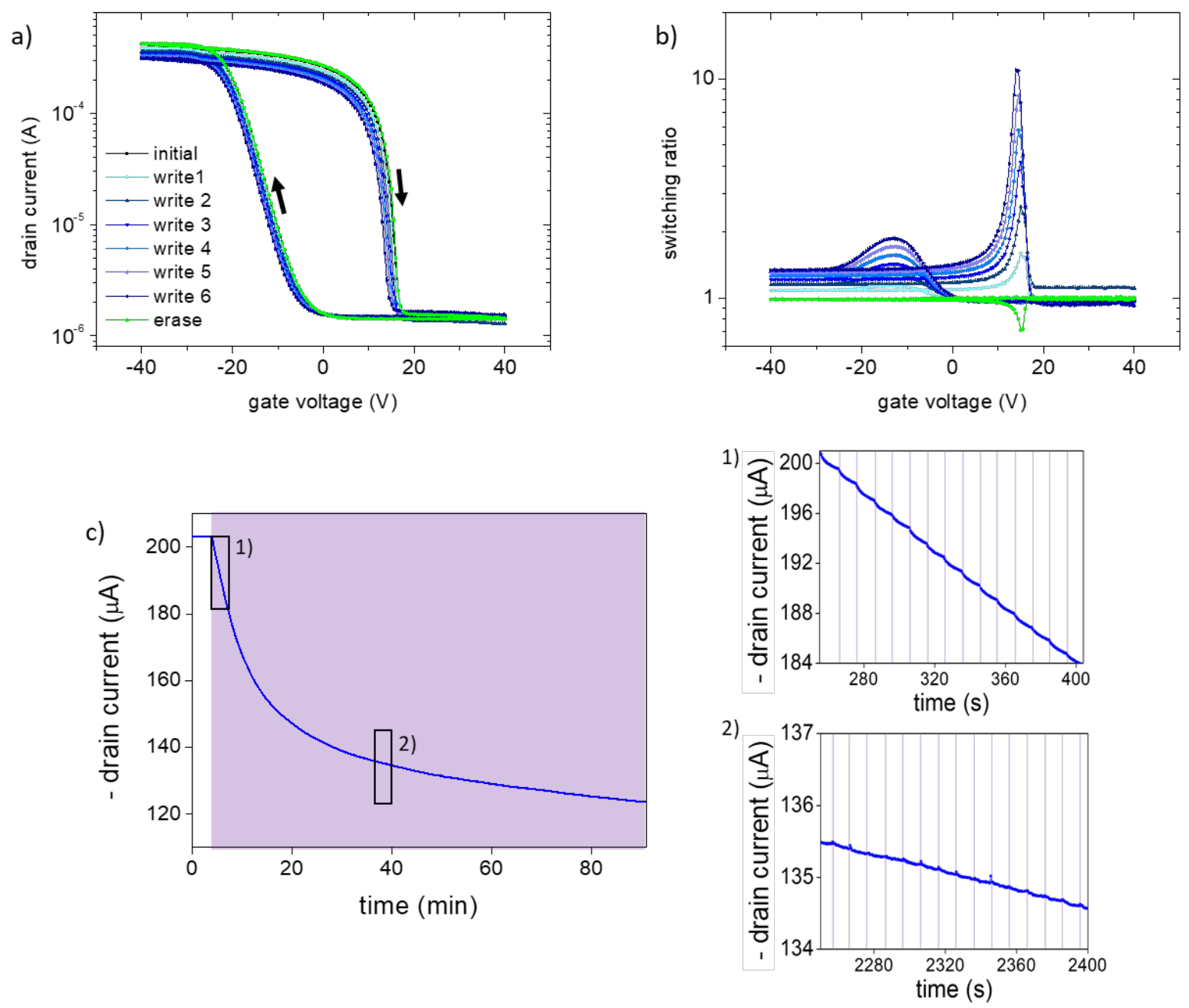

Figure 3: Light response of in P3HT/DAE-Me OSFeOFET. a) Full polarization transfer curves related to 6 writing steps using UV light ( $310 \mathrm{~nm}$ for $2 \mathrm{~min}$ ) and 1 erase step using visible light (530 $\mathrm{nm}$ for $5 \mathrm{~min}$ ). Please note that drain current is a hole current and, as such, its sign is negative. As a logarithmic scale is used to emphasize the current change over orders of magnitude, the absolute value of $\mathrm{I}_{\mathrm{D}}$ was used $\mathrm{b}$ ) Switching ratio ( $\left.\mathrm{I}_{\mathrm{Ds}, \mathrm{DAE}-\mathrm{Me} \_} / \mathrm{I}_{\mathrm{DS}, \mathrm{DAE}-\mathrm{Me} \_\mathrm{C}}\right)$ for each irradiation step as function of gate voltage (refer to panel a for the legend). c) Dynamic drain current response to $3 \mathrm{~ns} U V\left(310 \mathrm{~nm}\right.$ ) laser pulses (with incident power $\mathrm{P}_{\mathrm{i}}<1 \mu \mathrm{J} \mathrm{cm} \mathrm{c} \mathrm{s}^{-1}$, a light pulse is applied every $0.1 \mathrm{~Hz}$ ) when the ferroelectric layer is fully polarized. Given their short duration ( $3 \mathrm{~ns}$ ), illumination steps are not represented to scale. Insets of c panel showing the $3 \mathrm{~ns}$ induced steps at different times. After $40 \mathrm{~min}$ almost all DAE-Me is in its closed form, thus each 3 ns step induces markedly smaller current drop than at the beginning. Curves were plotted at $\mathrm{V}_{\mathrm{GS}}=0 \mathrm{~V}$ and $\mathrm{V}_{\mathrm{DS}}=-0.5 \mathrm{~V}$.

Besides storage capacity, speed is an integral part of all memory devices. In particular, the speed of read, write, and erase operations that can be achieved in a single memory element 


\section{WILEY-VCH}

is of utmost importance. In our memory cell, write and erase are linked both to the time required for ferroelectric polarization switching and for DAE isomerization. The ferroelectric switching time is of the order of fractions of milliseconds, as already extensively discussed previously. ${ }^{[22]}$ Here, therefore, we will mainly focus on the timescales for light programming. The isomerization times are an intrinsic properties of the DAE molecules and they cannot be considered the bottleneck for write operations since they have been reported to take place at picosecond (closure) and nanosecond to sub-nanosecond (opening) timescales, respectively. ${ }^{[23]}$ Therefore, in addition to the duration of the write pulse (being as short as $3 \mathrm{~ns}$ ), the overall programming time must take into account also the post-excitation charge-relaxation time. In order to determine how fast the memory might be operated around each polarization state, we monitored the current variation by performing time-resolved measurements during optical switching. The observed transient behavior is presented in Supplementary Figure S8, illustrating the switching dynamics and the speed of our memory cell. From this analysis, the post-excitation charge-relaxation time results in a minimum write-read delay on the order of $20 \mathrm{~ms}$ allowing a memory operational speed up to $50 \mathrm{~Hz}$. Further details of this analysis are presented in the Supplementary Materials. Alongside write/erase timing, the read operation also affects the device performances. In OSFeOFET, the readout relies on the current level since information is encoded in the amount of charges transmitted through the channel. Hence, the readout can be performed on the hundreds of millisecond timescale. Both the post-excitation charge-relaxation time and the readout time are crucially dependent on the device geometry, which determines the duration of the displacement current of non-equilibrium changes induced by illumination or applied voltage. ${ }^{[24]}$ The duration is mainly determined by the time-of-flight, which is the time required for charge carriers to drift across the transistor channel. ${ }^{[25]}$ In order to speed-up access to the memory, this time can be further reduced by decreasing the channel length. Beside increasing the memory speed, shorter channel length would allow for the 


\section{WILEY-VCH}

reduction of memory physical dimension which would enable the storage of more memory elements per unit area.

Finally, to demonstrate the full potential of our memory device for future high-density data storage we show that it is capable of reversible multi-level storage in a single cell, using simple but extremely efficient light write/erase cycles for each polarization state. Optical write/erase pulses of varying pulse energy enable us to freely move the current response between intermediate levels (defined by electrical program operation) with high reliability. This multi-level operation relies on the partial polarization states of PVDF-TrFE as base levels for intermediate conductivity states due to the consequential closure of DAE molecules. These mixed states exhibit current values in between those induced by the ferroelectric partial polarization shown in Figure 4.

a)

b)

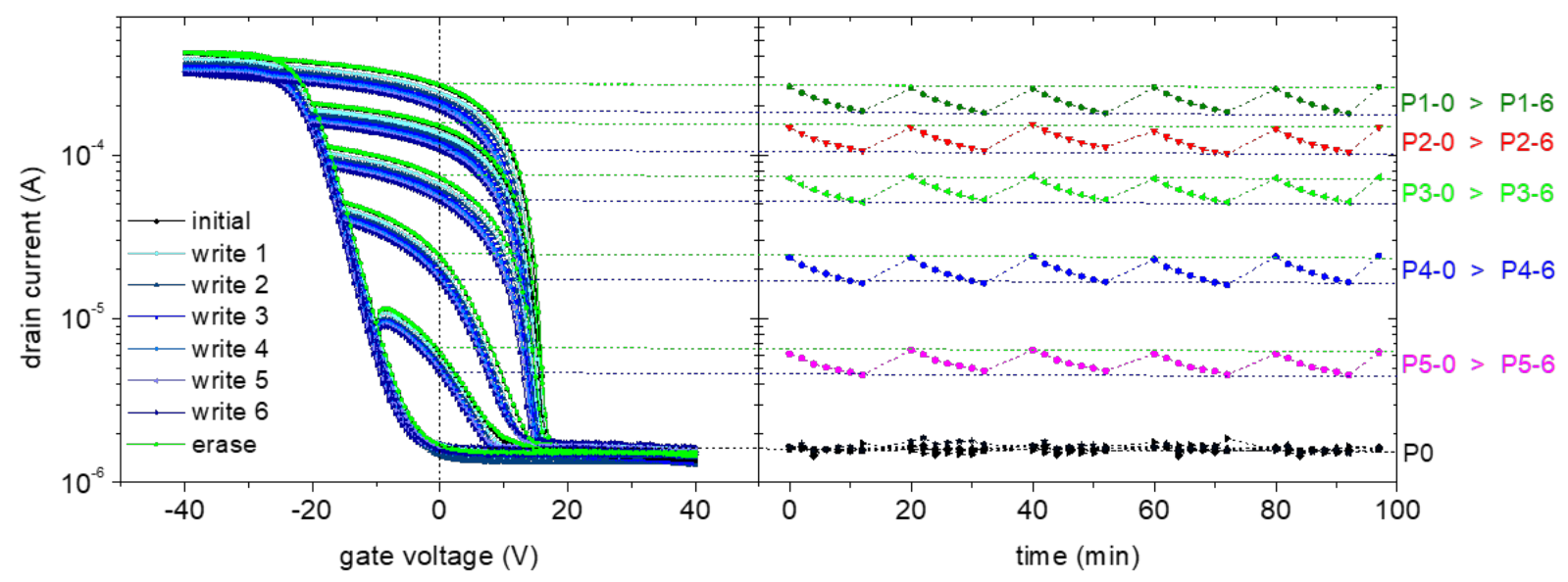

Figure 4: Current modulation in P3HT/DAE-Me OSFeOFET induced by light irradiations on each of the five polarization states. a) Light modulation of $\mathrm{IDS}_{\mathrm{DSS}}$ curves for 5 ferroelectric polarizations. Writing steps make use of UV light (310 $\mathrm{nm}$ for $2 \mathrm{~min}$ ), while erase step uses vis light $(530 \mathrm{~nm}$ for $5 \mathrm{~min})$. b) Dynamic drain current modulation to $\mathrm{P} / \mathrm{E}$ steps for each ferroelectric state showing reproducibility over 5 subsequent write erase cycles. [Please note that drain current is a hole current and, as such, its sign is negative. As a logarithmic scale is used to emphasize the current change over orders of magnitude, the absolute value of $I_{D}$ was used] 


\section{WILEY-VCH}

Six distinguishable levels are reached with 2 min UV pulses starting from each main polarization state. These levels were reached in a serial manner and subsequently the erase operation was carried out from the 6-level to the initial state. These results demonstrate that each level can be reached from both directions, that is, with a ring closure as well as an opening step. This implies that any level is accessible from all others, with very accurate control of the current levels and remarkable reliability, just by applying the appropriate electrical or optical write / erase sequence.

In summary, we have demonstrated the first multiresponsive organic device based on a photoswitchable-ferroelectric, non-volatile OSFeOFET memory that provides multi-level storage in a single transistor. Our approach uses a ferroelectric gate insulator integrated with photoswitchable molecules embedded in a semiconducting polymer matrix. The memory elements are switched between memory states by different stimuli: light pulses or electric field, which results in two independent ways to write or erase data. We have shown that using these programming operations, we were able to switch readily and directly the memory between multiple current levels, with accurate control of the readout signal and excellent repeatability. Moreover, we have demonstrated the capability for relatively fast (milliseconds), low light power intensity $\left(<1 \mu \mathrm{J} \mathrm{cm}^{-2} \mathrm{~s}^{-1}\right)$ optical write operations of the memory state while maintaining high readout SNR.

We observed that the readout in OSFeOFET can be performed on millisecond timescales, which could be further reduced by decreasing the channel length of the transistor. Consequently, in addition to increasing the memory speed, the memory physical dimension could also be reduced, which would enable the storage of more memory elements per unit area. The number of memory levels can be defined by tailoring the amount of DAE (wt\%) in the blend and the degree of $\beta$-phase crystallization of PVDF-TrFE within a single cell. The number of possible levels in a memory cell is limited by the separation between the highest and lowest current state for a certain partial polarization state and the required confidence interval of an intermediate 


\section{WILEY-VCH}

photo-induced level. The former can be increased by using either a ferroelectric material exhibiting higher remnant polarization or semiconducting polymer with higher mobility. The confidence interval, on the other hand, is mainly limited by the minor variations in the switching and by the SNR of the readout measurement. On the one hand, the number of memory levels can therefore be increased by using a higher readout drain voltage, ensuring a better SNR. On the other hand, as discussed above, a higher drain voltage applied during the read operation would be detrimental for the data retention. Therefore, the right trade-off between these competing factors has to be identified and one example of such multi-level operation is demonstrated in Figure 4.

Our ferroelectric-photoswitchable system is fully scalable: the photoswitching units are just few nm long, they can also be switched individually ${ }^{[26]}$ and PVDF-TrFE organic ferroelectric devices have seen an interesting scaling trend in the switching time and the switching voltage as a function of the device area. ${ }^{[27]}$ All these attributes, which are essential for the realization of a high-performance memory device, are also fully compatible with flexible substrates. Therefore, these very first experimental results demonstrate the higher performance of OSFeOFET in term of storage capacity and data retention in comparison with existing multicomponent organic memories and they establish the foundations for promising devices in multifunctional opto-electronic application. Our OSFeOFET may become a key element in neuromorphic devices and synaptic arrays to fulfill the current and future needs of increasingly demanding computational density which cannot be met by scaling down complementary metaloxide-semiconductor (CMOS) transistors.

\section{Supporting Information}

Supporting Information is available from the Wiley Online Library or from the author.

\section{Acknowledgements}




\section{WILEY-VCH}

This work was financially supported by EC through the Marie Sklodowska-Curie ITN project iSwitch (GA-642196), the ERC projects SUPRAFUNCTION (GA-257305) and LIGHT4FUNCTION (GA-308117), the Labex projects CSC (ANR-10-LABX-0026 CSC) and NIE (ANR-11-LABX-0058 NIE) within the Investissement d'Avenir program ANR-10-IDEX0002-02, the International Center for Frontier Research in Chemistry (icFRC), Institut Universitaire de France (IUF), the Natural Sciences and Engineering Research Council of Canada (NSERC) through an individual Discovery Grant and the German Research Foundation (DFG via SFB 658 and SFB 951), and the Slovenian Research Agency under the research core funding No. P1-0055.

Received: ((will be filled in by the editorial staff))

Revised: ((will be filled in by the editorial staff)) Published online: ((will be filled in by the editorial staff))

\section{Conflict of Interest}

The authors declare no conflict of interest.

\section{References}

[1] a) W. Zhang, R. Mazzarello, M. Wuttig, E. Ma, Nat Rev Mater 2019, 4, 150; b) K. Asadi, D. M. De Leeuw, B. De Boer, P. W. Blom, Nat Mater 2008, 7, 547; c) Y.-H. Chu, L. W. Martin, M. B. Holcomb, M. Gajek, S.-J. Han, Q. He, N. Balke, C.-H. Yang, D. Lee, W. Hu, Nat Mater 2008, 7, 478; d) N. A. Spaldin, R. Ramesh, Nat Mater 2019, 18, 203; e) S. Bertolazzi, P. Bondavalli, S. Roche, T. San, S. Y. Choi, L. Colombo, F. Bonaccorso, P. Samorì, Adv Mater 2019, 31, 1806663; f) S. S. Parkin, M. Hayashi, L. Thomas, Science 2008, 320, 190.

[2] J. E. Green, J. W. Choi, A. Boukai, Y. Bunimovich, E. Johnston-Halperin, E. DeIonno, Y. Luo, B. A. Sheriff, K. Xu, Y. S. Shin, Nature 2007, 445, 414.

[3] S. K. Hwang, I. Bae, R. H. Kim, C. Park, Adv Mater 2012, 24, 5910.

[4] a) P. Heremans, G. H. Gelinck, R. Muller, K.-J. Baeg, D.-Y. Kim, Y.-Y. Noh, Chem Mater 2010, 23, 341; b) C. Ma, Z. Luo, W. Huang, L. Zhao, Q. Chen, Y. Lin, X. Liu, Z. Chen, C. Liu, H. Sun, Nat Commun 2020, 11, 1439.

[5] a) M. Gu, X. Li, Y. Cao, Light Sci Appl 2014, 3, e177; b) M. L. Lalieu, R. Lavrijsen, B. Koopmans, Nat Commun 2019, 10, 110.

[6] a) G. W. Burr, B. N. Kurdi, J. C. Scott, C. H. Lam, K. Gopalakrishnan, R. S. Shenoy, IBM J Res Dev 2008, 52, 449; b) J. Ouyang, C.-W. Chu, C. R. Szmanda, L. Ma, Y. Yang, Nat Mater 2004, 3, 918; c) J. C. Scott, L. D. Bozano, Adv Mater 2007, 19, 1452.

[7] a) Q.-D. Ling, D.-J. Liaw, C. Zhu, D. S.-H. Chan, E.-T. Kang, K.-G. Neoh, Prog Polym Sci 2008, 33, 917; b) S. Möller, C. Perlov, W. Jackson, C. Taussig, S. R. Forrest, Nature 2003, $426,166$.

[8] H. Ling, K. Tan, Q. Fang, X. Xu, H. Chen, W. Li, Y. Liu, L. Wang, M. Yi, R. Huang, Adv Electron Mater 2017, 3, 1600416. 


\section{WILEY-VCH}

[9] a) M. Herder, F. Eisenreich, A. Bonasera, A. Grafl, L. Grubert, M. Pätzel, J. Schwarz, S. Hecht, Chem Eur J 2017, 23, 3743.

[10] W. Y. Kim, H.-D. Kim, T.-T. Kim, H.-S. Park, K. Lee, H. J. Choi, S. H. Lee, J. Son, N. Park, B. Min, Nat Commun 2016, 7, 10429.

[11] H. Zhu, C. Fu, M. Mitsuishi, Polym Int 2020, DOI: 10.1002/pi.6029.

[12] D. Zhao, I. Katsouras, M. Li, K. Asadi, J. Tsurumi, G. Glasser, J. Takeya, P. W. Blom, D. M. De Leeuw, Sci Rep 2014, 4, 5075.

[13] R. C. Naber, K. Asadi, P. W. Blom, D. M. de Leeuw, B. de Boer, Adv Mater 2010, 22, 933.

[14] a) K. Asadi, P. W. Blom, D. M. de Leeuw, Appl Phys Lett 2011, 99, 156; b) B. Kam, X. Li, C. Cristoferi, E. C. Smits, A. Mityashin, S. Schols, J. Genoe, G. Gelinck, P. Heremans, Appl Phys Lett 2012, 101, 033304; c) D. Zhao, I. Katsouras, K. Asadi, W. A. Groen, P. W. Blom, D. M. de Leeuw, Appl Phys Lett 2016, 108, 232907.

[15] T. Leydecker, M. Herder, E. Pavlica, G. Bratina, S. Hecht, E. Orgiu, P. Samorì, Nat Nanotechnol 2016, 11, 769 .

[16] a) D. Setiadi, P. Regtien, P. Sarro, Sens Actuator A Phys 1994, 42, 585; b) M. I. Akcan, C. Topacli, Polym Int 2001, 50, 835; c) R. Naber, M. Mulder, B. De Boer, P. Blom, D. De Leeuw, Org Electron 2006, 7, 132; d) W.-H. Kim, J.-H. Bae, M.-H. Kim, C.-M. Keum, J. Park, S.-D. Lee, J Appl Phys 2011, 109, 024508.

[17] D. Singh, A. Garg, Phys Chem Chem Phys 2017, 19, 7743.

[18] a) J.-W. Yoon, S.-M. Yoon, H. Ishiwara, Jpn J Appl Phys 2010, 49, 030201; b) M. A. Khan, H. N. Alshareef, I. N. Odeh, M. N. Almadhoun, US Patents 2018 (Patent No.: US 10,096,352 B2); c) T. Ishida, US Patents 2009 (Patent No.: US 2009/0294817 A1); d) H. Xu, J. Zhong, X. Liu, J. Chen, D. Shen, Appl Phys Lett 2007, 90, 092903; e) M. Li, N. Stingelin, J. J. Michels, M.-J. Spijkman, K. Asadi, K. Feldman, P. W. Blom, D. M. de Leeuw, Macromolecules 2012, 45, 7477.

[19] P. Larsen, R. Cuppens, G. Spierings, Ferroelectrics 1992, 128, 265.

[20] a) L. Hou, T. Leydecker, X. Zhang, W. Rekab, M. Herder, C. Cendra, S. Hecht, I. McCulloch, A. Salleo, E. Orgiu, P. Samorì, J Am Chem Soc 2020, 142, 11050; b) E. Orgiu, N. Crivillers, M. Herder, L. Grubert, M. Pätzel, J. Frisch, E. Pavlica, D. T. Duong, G. Bratina, A. Salleo, N. Koch, S. Hecht, P. Samorì, Nat Chem 2012, 4, 675.

[21] M. Herder, B. M. Schmidt, L. Grubert, M. Pätzel, J. Schwarz, S. Hecht, J Am Chem Soc 2015, 137, 2738.

[22] R. C. G. Naber, C. Tanase, P. W. M. Blom, G. H. Gelinck, A. W. Marsman, F. J. Touwslager, S. Setayesh, D. M. de Leeuw, Nat Mater 2005, 4, 243.

[23] M. Irie, T. Fulcaminato, K. Matsuda, S. Kobatake, Chem Rev 2014, 114, 12174.

[24] E. Pavlica, G. Bratina, J Phys D Appl Phys. 2008, 41, 135109.

[25] a) L. Dunn, D. Basu, L. Wang, A. Dodabalapur, Appl Phys Lett 2006, 88, 063507; b) F. Otón, R. Pfattner, E. Pavlica, Y. Olivier, E. Moreno, J. Puigdollers, G. Bratina, J. Cornil, X. Fontrodona, M. Mas-Torrent, J. Veciana, C. Rovira, Chem Mater 2011, $23,851$.

[26] C. C. Jia, A. Migliore, N. Xin, S. Y. Huang, J. Y. Wang, Q. Yang, S. P. Wang, H. L. Chen, D. M. Wang, B. Y. Feng, Z. R. Liu, G. Y. Zhang, D. H. Qu, H. Tian, M. A. Ratner, H. Q. Xu, A. Nitzan, X. F. Guo, Science 2016, 352, 1443.

[27] S. Das, J. Appenzeller, Org Electron 2012, 13, 3326. 